\title{
Coincidence of Upper Eyelid Coloboma with Posterior Synechia in a Cat: A Case Report
}

\section{Fatemeh Etemadi', Ramin Mazaheri Nezhad Fard ${ }^{2}$, Nagha Tamimi ${ }^{3}$, Mahdieh Rezaei ${ }^{3}$, Seyed Milad Vahedi', Hannaneh Golshahi ${ }^{4}$ and Seyed} Javid Aldavood ${ }^{3 *}$

${ }^{1}$ Faculty of Veterinary Medicine, University of Tehran, Tehran, Iran

${ }^{2}$ RastegarReference Laboratory, Faculty of Veterinary Medicine, University of Tehran, Tehran, Iran

${ }^{3}$ Department of Clinical Sciences, Faculty of Veterinary Medicine, University of Tehran, Tehran, Iran

${ }^{4}$ Department of Pathology, Faculty of Veterinary Medicine, University of Tehran, Tehran, Iran

\begin{abstract}
Coloboma is a genetic disorder which occurs due to a defect in the eye structure such as iris, lens, choroid plexus or optic disc. In this report, coloboma with posterior synechia in the right eye and iris prolapse in the left eye of a kitten are reported. A two-month-old female domestic short-hair cat with discrete from eyes, hyperemia, purulent discharge and photophobia was referred to the Small Animal Teaching Hospital of the Faculty of Veterinary Medicine, University of Tehran. In clinical examination of the eyes, the lack of lateral part of the upper eyelid and posterior synechia in the right eye and iris prolapse in the left eye were found. Bacterial conjunctivitis was detected as well. The kitten case history showed a hereditary reason for the right eye deficiency and a traumatic reason for the left eye iris prolapse. Eye examination revealed no other hereditary disorders. Because of the limited disorder of the right eyelid, treatment was based on topical antibiotic and anti-inflammation drugs. Surgery was performed to treat the left eye iris prolapse. The present case is the first report of coincidence of upper eyelid coloboma with posterior synechia in cat in veterinary literature.
\end{abstract}

Keywords: Coloboma; Synechia; Iris Prolapse; Cat; Eye; Iran

\section{Introduction}

One of the congenital anomalies of ocular tissue is coloboma. Ocular coloboma usually results from defective closure of the embryonic fissure [1]. Most frequently this fissure is occurred in the optic disc (slightly ventral and medial to the optic nerve), however it can be located in the iris or ciliary body $[1,2]$. This disorder can be diagnosed in the first semester of pregnancy during the eyes formation [3]. Eyelid coloboma can be unilateral or bilateral, symmetrical orassymetrical, associated with other ocular anomalies such asmicrophthalmia, persistent pupillary membrane, choroidal and optic nerve colobomas, retinal dysplasia and dermoids [1,4]. Feline ocular coloboma is a relatively well documented lesion and occurs sporadically in both domestic and larger cats [4]. Several etiologies have been suggested for this disorder, they are: heritable trait, abnormal developmental differentiation of eyelid structures and teratogenicity [4]. Colobomasmay cause congenital or progressive blindness or maybe clinically in apparent for life [1,5]. Eyelid coloboma causes significant discomfort. Clinical signs such as blepharospasm, epiphora, irregular corneal surface resulting from trichiasis [6]. According to author's knowledge, the present case is the first report of coincidence of upper eyelid coloboma with posterior synechia in cat in veterinary literature.

\section{Case Description}

A two-month-old female domestic short-hair cat with discrete from eyes, hyperemia and purulent discharge and photophobia was referred to the Small Animal Teaching Hospital of the Faculty of Veterinary Medicine, University of Tehran. Ophthalmology examination revealed the lack of lateral part of the upper eyelid and posterior synechia in the right eye and iris prolapse in the left eye which was possibly the actual cause of the cat's bacterial conjunctivitis (Figure 1). The kitten case history showed a hereditary reason for the right eye defect and a traumatic reason for the left eye deformity. Examination of the eyes did not reveal any other hereditary diseases.

\section{Discussion}

In general, eyelid defects in cats may result from trauma, surgical removal of masses, or colobomas [4]. Eyelid coloboma has been reported in humans, cats, dogs, sheep, horses, Texas cougar and snow leopard, Charolais cattle, rats, mice and rabbits [6-8]. This disorder tends to be

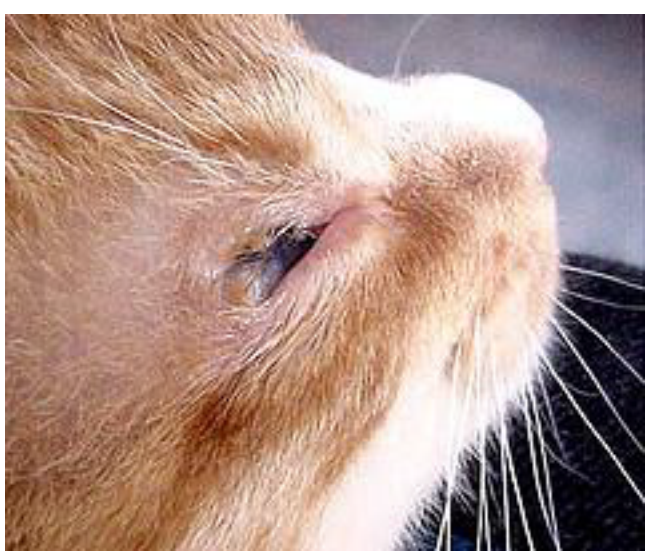

Figure 1: Coloboma in the right eye of a kitten.

*Corresponding author: Seyed Javid Aldavood, Department of Clinical Sciences, Faculty of Veterinary Medicine, University of Tehran, Tehran, Iran, Tel: +98-21 66920035; Fax: +98-2166933222; E-mail: sja@ut.ac.ir

Received June 24, 2013; Accepted July 17, 2013; Published July 19, 2013

Citation: Etemadi F, Fard RMN, Tamimi N, Rezaei M, Vahedi SM, et al. (2013) Coincidence of Upper Eyelid Coloboma with Posterior Synechia in a Cat: A Case Report. J Veterinar Sci Technol 4: 139. doi:10.4172/2157-7579.1000139

Copyright: (c) 2013 Etemadi F, et al. This is an open-access article distributed under the terms of the Creative Commons Attribution License, which permits unrestricted use, distribution, and reproduction in any medium, provided the original author and source are credited. 
bilateral and essential lesion involves complete or partial eyelid agenesis (usually lack of lateral part of the upper eyelids) [4,6,9]. In the present case, agenesis of lateral part of the upper eyelid was diagnosed, however unilateral coloboma was existed. In humans, colobomas are located nasally in the upper lid and the laterally in the lower eyelid [6].

The exact etiology of coloboma has not been established yet, however, heritable traits contributing to abnormal development when optic fissure closing and differentiation of eyelid structures and teratogenicity are the suspected reasons $[4,6]$.

Colobomas may result from asynchronous development of the retinal and choroid layers, or focal dysplasia of the retina with failure to induce the overlying choroid. Neural crest cells are responsible for coat color and formation of certain connective tissues, like the sclera and choroid of the eye. Thus an abnormality of neural crest cells can produce ocular anomalies in color-dilution breeds. However, normalpigmented animals may have a sporadic form of coloboma [1], that this condition was in agreement with our case.

Although in feline coloboma, some sporadic cases of coloboma have been reported in the domestic short haired, Persian and Burmese, the breed predisposition for feline eyelid coloboma has not been documented yet $[6,7]$. The present case was a domestic short haired cat.

This disorder may occur as a syndrome in a litter or other concurrent congenital ocular defects like persistent pupillary membrane, microphthalmia, congenital anterior synechia, choroidal and optic nerve colobomas, retinal dysplasia and dermoids $[4,6,9]$. In our case, coincidence of upper eyelid coloboma with posterior synechia was diagnosed.

Eyelid colobomas may be medically managed until the patient is old enough to undergo surgery with the surgical technique [4]. Several surgical techniques have been described to repair the upper eyelids, ranging from simple closure of smaller colobomas to surgical procedures that move skin and conjunctiva into the defect [8]. Small lesions (1/4-1/3 lid margin) can be repaired by simple closure (with or without a releasing incision at the lateralcanthus), in larger lesions (1/3 margin or greater) more complex surgical techniques must be used. Techniques described include Roberts' and Bistners' pedicle flap and its modification by Dziecyc \& Millichamp, the rotating flap, the Z-flap, the semicircular flap, the bucket-handle or Cutler-Beard procedure, and the Mustardé or 'cross-lid' technique [4] in addition, subdermal injection of collagen had been used for replacing the bulk of missing eyelid [6]. In the current report, because of a limited disorder in the right eyelid of the kitten, treatment was only based on topical antibiotics and anti-inflammation drugs. Surgery was later performed to treat the iris prolapse within the kitten's left eye.

To conclude this paper report coincidence of upper eyelid coloboma with posterior synechia in cat for first time in veterinary literature.

\section{References}

1. Schuh JC, Ferguson JG, Fisher MA (1991) Congenital coloboma in a llama. Can Vet J 32: 432-433.

2. Wilcock BP (1983) Ocular anomalies. In, Peiffer RL (Ed): Comparative Ophthalmic Pathology. 3-46, Springfield, Charles C, Thomas.

3. Occelli L, Neaderland M (2011) Reconstruction of an iatrogenic eyelid defect in a Bichon Frise. Pratique Medicaleet Chirurgicale de IAnimal de Compagnie 46: 99-105.

4. Esson D (2001) A modification of the Mustardé technique for the surgical repair of a large feline eyelid coloboma. Vet Ophthalmol 4: 159-160.

5. Schuh JC (1989) Bilateral colobomas in a horse. J Comp Pathol 100: 331-335

6. Cheng SH, Yeh LS, Lin CT (2006) Case report: Eyelid coloboma in a domestic short haired Cat. Taiwan Vet J 32: 265-270.

7. Cutler TJ (2002) Bilateral eyelid agenesis repair in a captive Texas cougar. Vet Ophthalmol 5: 143-148.

8. Wolfer JC (2002) Correction of eyelid coloboma in four cats using subdermal collagen and a modified Stades technique. Vet Ophthalmol 5: 269-272.

9. Barnett K, Crispin S (1989) In: Feline Ophthalmology: an Atlas and Text. W.B. Saunders Co, London, UK. 